5. - Bericht über neuere Untersuchungen und Probleme aus der Theorie der algebraischen Zahlkörper, Teil I, Jahresbericht der Deutschen Mathematiker-Vereinigung vol. 35 (1926) pp. 1-55.

6. Julia Robinson, Definability and decision problems in arithmetic, J. Symb. Logic vol. 14 (1949) pp. 98-114.

7. R. M. Robinson, Undecidable rings, Trans. Amer. Math. Soc. vol. 70 (1951) pp. 137-159.

8. - Arithmetical definability of field elements, J. Symb. Logic vol. 16 (1951) pp. $125-126$.

9. A. Tarski, A. Mostowski, and R. M. Robinson, Undecidable theories, Amsterdam, North-Holland Publishing Company, 1953.

10. Ernst Witt, Theorie der quadratischen Formen in beliebigen Körpern, J. Reine Angew. Math. vol. 176 (1936) pp. 31-44.

Contra Costa County, California

\title{
CONCERNING LOCAL SEPARABILITY IN LOCALLY PERIPHERALLY SEPARABLE SPACES
}

\section{B. TREYBIG}

Alexandroff [1] has shown that a connected metric space is completely separable if it is locally completely separable. In the previous statement "completely separable" may be replaced with "separable," since these are equivalent conditions in a metric space. In his dissertation (Texas, 1958) the author has shown an example of a connected, locally peripherally separable [2], metric space which is not separable, but which has the property that the set of all points at which it is not locally separable is separable. The purpose of this paper is to give a further result in this direction.

Theorem. If $\Sigma$ is any connected, locally peripherally separable, metric space which is not separable, then the set of all points at which $\Sigma$ is not locally separable is uncountable.

Proof. On the contrary, suppose that there exists such a space $\Sigma$ where the set $M$ of all points at which $\Sigma$ is not locally separable is countable. Let $M$ be denoted by $P_{1}+P_{2}+P_{3}+\cdots$, where, if $i \neq j$, $P_{i} \neq P_{j}$. For each positive integer $n$ let $G_{n}$ be the collection of all locally peripherally separable domains having diameter less than $1 / n$. Let $d$ denote a positive integer and $g_{1}, g_{2}, g_{3}, \ldots$ denote a sequence such that for each $n, P_{n}$ and $g_{n}$ are elements of $g_{n}$ and $G_{n+d}$, respectively. Let $H$ be a collection to which $x$ belongs if and only if $x$ is $g_{i}$ for some $i$. For each positive integer $n$, let $G_{n}^{\prime}$ be the collection of all 1959.

Presented to the Society, August 29, 1958; received by the editors January 22, 
separable elements of $G_{n}$. The point set $M$ is closed because $\Sigma$ would not be locally separable at any limit point of $M$. For each point $P$ of $S-M$ let $j(P)$ denote the least integer $i$ such that some domain $r$ of $G_{d}^{\prime}$ has the property that if $x$ and $y$ are two intersecting domains of $G_{i}^{\prime}$ such that $x+y$ contains $P$, then $x+y$ is a subset of $r$. Let $r(P)$ denote some such $r$.

Suppose that no countable subcollection of $G_{d}$ covers $S$. Each point on the boundary of $H^{*}$ lies on the boundary of $g_{i}$ for some $i$; if not, $S$ would not be locally separable at such a point. Therefore $\bar{H}^{*}-H^{*}$ is separable. Let $Q_{1}$ denote some countable subcollection of $G_{d}^{\prime}$ which covers $\bar{H}^{*}-H^{*}$. Let $M_{1}=\bar{Q}_{1}^{*}+H^{*}$. Since $Q_{1}$ is countable and each domain of $Q_{1}$ is separable, $M_{1}-H^{*}$ is separable. There is a countable set $K_{1}$ dense in $M_{1}-H^{*}$ such that the set $Q_{2}$ of all $r(P)$ 's for $P^{\prime}$ 's in $K_{1}$ covers $M_{1}-H^{*}$. Let $M_{2}=M_{1}+\bar{Q}_{2}^{*}$. There exists a countable set $K_{2}$ dense in $M_{2}-H^{*}$ such that the set $Q_{3}$ of all $r(P)$ 's for $P$ 's in $K_{2}$ covers $M_{2}-H^{*}$. Let $M_{3}=M_{2}+\bar{Q}_{3}^{*}$ and consider a continuation of this process.

The collection $H+\left(Q_{1}+Q_{2}+Q_{3}+\cdots\right)$ is countable and therefore does not cover $S$. Clearly $\left[H+\left(Q_{1}+Q_{2}+Q_{3}+\cdots\right)\right]^{*}$ is a domain; so let $P$ denote some point on the boundary of this domain. Let $K=\left(K_{1}+K_{2}+\cdots\right)$. Let $R_{1}$ denote some domain of $G_{d}^{\prime}$ which contains $P$ and let $x_{1}$ denote the largest integer $i$ such that $R_{1}$ belongs to $G_{i}^{\prime}$. Let $T_{1}$ denote a point of $K$ in $R_{1}$. Let $R_{2}$ denote a domain of $G_{x_{1}+1}^{\prime}$ which contains $P$ and is a subset of $R_{1}-T_{1}$. Let $x_{2}$ denote the largest integer $i$ such that $R_{2}$ belongs to $G_{i}^{\prime}$ and let $T_{2}$ denote a point of $K$ in $R_{2}$. Let $R_{3}$ denote a domain of $G_{x_{2}+1}^{\prime}$ which contains $P$ and is a subset of $R_{2}-T_{2}$. Let $x_{3}$ denote the largest integer $i$ such that $R_{3}$ belongs to $G_{i}^{\prime}$, and so forth. $d \leqq x_{1}<x_{2}<x_{3}<\cdots$. For each $n$, $x_{n}>j\left(T_{n}\right)$; if not, for some $n, R_{n}$ would be a subset of $r\left(T_{n}\right)$ and $P$ would lie in $Q_{i}^{*}$ for some $i$. There exists a positive integer $t$ such that if $x$ and $y$ are two intersecting domains of $G_{x_{t}}^{\prime}$ such that $x+y$ intersects $R_{t}$, then $x+y$ is a subset of $R_{1}$. Therefore $j\left(T_{t}\right) \geqq x_{t}$. This yields a contradiction; so $H+\left(Q_{1}+Q_{2}+\cdots\right)$ covers $S$. Therefore for each $d$ some countable subcollection of $G_{d}$ covers $S . \Sigma$ is therefore separable; but this is contrary to the hypothesis, so $M$ is uncountable.

\section{REFERENCES}

1. Paul Alexandroff, Über die Metrization der im kleinen kompakten topologischen Raume, Math. Ann. vol. 92 (1924) pp. 294-301.

2. F. B. Jones, A theorem concerning locally peripherally separable spaces, Bull. Amer. Math. Soc. vol. 41 (1935) pp. 437-439.

The University of Texas AND

TUlane University 